\title{
Discovering of Cooperation Pattern Evolution Path in Tsinghua University: a Patent Cooperation Network Perspective
}

\author{
Qiong Liu \\ Institute of Scientific and Technical Information \\ Jiangsu University \\ Zhenjiang, China \\ liu123452@163.com
}

\begin{abstract}
This paper retrieves collaboration patents of Tsinghua University between 1993 and 2012, and draws the Evolvement Path. Based on the social network visualization analysis, applicant network between Tsinghua University and four different types of organizations are established to reflect the cooperation model and pathway between Tsinghua University and other organizations.
\end{abstract}

Keywords-Colleges and universities; Tsinghua University; Patent cooperation; Social network visualization; Evolvement Path

\section{INTRODUCTION}

Patent is the important part which transfers scientific knowledge into technology or products. Analyze the cooperation relationship and evolvement path to patent applicant of an industry or institute, which can help us to know its collaborative innovation rules. Based on applied patents and granted patents of Japanese college, $\mathrm{Hu}$ (2012) found that industry-university cooperation can reflect the basic relationship between development of modern science and technology and the modern economic growth. Liu (2013) selected the patent cooperation network of Integrated Circuit Industry to analyze the influence factors of knowledge diffusion. Ponomariov B (2013) studied the major feature of patent cooperation between American colleges and nanotechnology industry. James Simmie (2014) established the patent evolution path of the wind power industry. Liu (2012) built the patent technology network to find the evolution path of nanotechnology. Pen (2013) used the patent citation network to study technology evolution path of laser display industry. Janghyeok Yoon (2014) built the patent information network to track technology evolution trends of the printed electronics. Chan-Yuan Wong (2013) chose international patents and SCI papers in Japan and Korea as the research sample to analyze the innovation path of science and technology. It is shown from the above that patent cooperation network can be used to reflect the trend of industry development. However, there is very little research about building patent cooperation network by using of analysis of the cooperation models between colleges and other institutes.

The purpose of this paper is to analyze the cooperation patents of Tsinghua University from 1993 to 2012, which mine the cooperation model between Tsinghua University and other institutes.

\section{DATA}

To fulfill our research objectives we analyze a sample of Tsinghua university cooperation invention patents that are registered at the State Intellectual Property Office of China. For each patent, data about the title, abstract, grant date, references, as well as the name, city of inventors and firms are extracted. After cleaning for duplicates and missing data, our sample contains 2570 unique university cooperation invention patents obtained from 1992 to 2012. First, the patents divide into four stages: 1993-1999, 2000-2004, 2005-2009, and 2010-2012. Second, the cooperation institutes divide into three types: college, academic institutions, and enterprises. Third, use the social network visualization and NoteXL to build the patent cooperation with four stages and three institute types. At last, according to mining the information of cooperation patent applicants and patent subjects, track the cooperation models of Tsinghua University and other institutes. The information sources are website of Tsinghua University, cooperation institutes, NSTI and CNKI.

\section{PATENT COOPERATION GENERAL SITUATION}

Table 1 shows the patent cooperation situation between Tsinghua University and three types of institutes in four stages. We can see from Table 1 that there is few numbers applicant and application of cooperation patents, whatever type of organization from 1993 to 1999. Since 2000, the number of patents raises drastically, especially the cooperation with enterprises. In addition to macroscopic impact of Intellectual property and industry-university-research cooperation policy in China, Tsinghua University has made complete management system and regulations in intellectual property protection and industry-university-research cooperation. In 1996, Tsinghua University developed several rules to protect intellectual property. To systematically manage the strategic cooperation with internation, central government, local which can promote the cooperative innovation between organizations in real time, Tsinghua University has set up the intellectual property management office that it formed five different cooperation models: local academic institute, university-enterprise 
Cooperation committee, the integration office, fund model and joint research institution base on provincial-school framework cooperation, college-enterprise cooperation and project cooperation.

TABLE I. PATENT COOPERATION SITUATION OF TSINGHUA UNIVERSITY

\begin{tabular}{|c|l|c|c|c|}
\hline & & $\begin{array}{c}\text { Tsinghua } \\
\text { Uni-College }\end{array}$ & $\begin{array}{c}\text { Tsinghua Uni- } \\
\text { Academic } \\
\text { institutions }\end{array}$ & $\begin{array}{c}\text { Tsinghua } \\
\text { Uni- } \\
\text { enterprise }\end{array}$ \\
\hline \multirow{2}{*}{$\begin{array}{c}1993- \\
1999\end{array}$} & Applicant & 2 & 10 & 21 \\
\cline { 2 - 5 } & Application & 2 & 10 & 26 \\
\hline \multirow{2}{*}{$2000-$} & Applicant & 8 & 19 & 86 \\
\cline { 2 - 5 } 2004 & Application & 12 & 37 & 294 \\
\hline \multirow{2}{*}{$2005-$} & Applicant & 18 & 37 & 157 \\
\cline { 2 - 5 } 2009 & Application & 44 & 62 & 1009 \\
\hline \multirow{2}{*}{$2010-$} & Applicant & 15 & 38 & 946 \\
\cline { 2 - 5 } 2012 & Application & 41 & 88 & 183 \\
\hline
\end{tabular}

\section{TSINGHUA UNIVERSITY-COLLEGE COOPERATION} EVOLUTION PATH

Fig. 1 shows the patent cooperation situation with Tsinghua University and college in four stages. There is two colleges cooperation with Tsinghua University before 2000. China University of Geosciences has all along maintained academic exchanges with Tsinghua University, because China University of Geosciences, was formerly formed by corresponding department of Peking University, Tsinghua University, Tianjin University, and Tangshan Railway College. The cooperation patent topic is vacuum melting composite coating cylinder liner and the inventor is Chengbiao Wang and Shizhu Wen. Through the information mining, Chengbiao Wang was master and doctor of department of precision instruments, Tsinghua University, whose supervisor is Shizhu Wen. It can be seen that original cooperation between Tsinghua University and other colleges generated talent exchange, which is the traditional college-college cooperation model.

From 2000, cooperate between Tsinghua University with colleges is frequent. There are 10 cooperation colleges and 34 patents. Hong Kong Baptist University, Nankai University and Beihang University account for the largest share. Noteworthy is the Hong Kong Baptist University established the Whalen academic exchange center with Tsinghua University in 1999. Establisment of exchange center break through the short-term cooperation model, which promotes talent cultivation and academic exchange between the two places. At present it has become the important base of scientific research and exploring new technology.

Tsinghua University cooperated with 15 colleges to apply for 24 patents from 2005 to 2009. We can see from Fig. 1 that patent cooperation between Nankai University and Tsinghua University have continued since 2000 because Zihe Rao who is the president of Nankai University and vice President of the institute of life science and medicine of Tsinghua University built joint laboratory of Nankai University, Tsinghua University, Institute of Biophysics of Chinese Academy of Sciences. Otherwise, the most of Cooperation college is University of Science and Technology Beijing. The cooperation model is senior talent cooperation and communication. For example, Professor Henghu Sun of Tsinghua University and Professor Wen Ni of University of Science and Technology Beijing are Nanotechnology experts, and they keep long-term academic cooperation and technology exchange.

Tsinghua University cooperated with 14 colleges to apply for 28 patents since 2010. Some colleges including University of Science and Technology Beijing, Beihang University, Beijing University of Technology, Beijing University of Chemical Industry and Guilin University of Electronic Science and Technology keep long-term patent cooperation and form closely talent network. At that time, the most of cooperation derived from the participation of the third party. For example, 63963 troops of the Chinese People's Liberation Army cooperate with Tsinghua University and University of Science and Technology Beijing for the development of automatic identification of vehicle instrument technology.

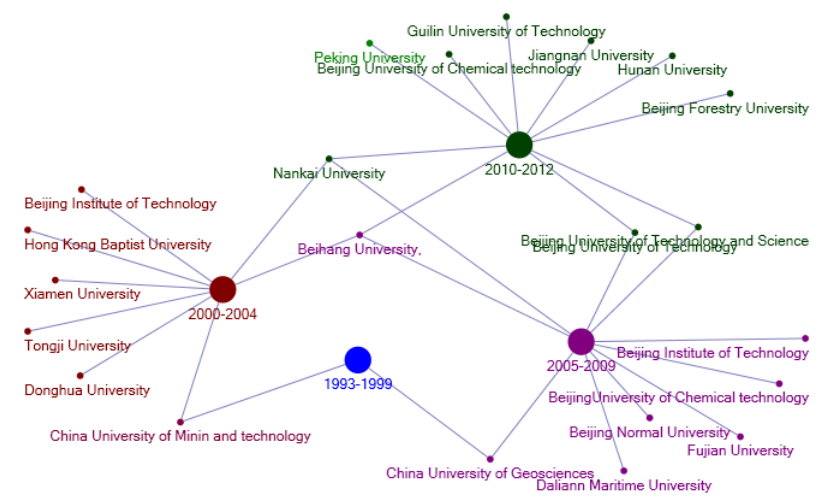

FIGURE I. Tsinghua University-College cooperation evolution path

\section{TSINGHUA UNIVERSITY- ACADEMIC INSTITUTIONS COOPERATION EVOLUTION PATH}

Fig. 2 shows the patent cooperation situation between Tsinghua University and the top 10 academic institutions in the fourth stage. It is seen from Fig. 2 that there are many cooperation institutes before 2000, but the number of patents is just only one. The cooperation model is development of national projects. For instance, Tsinghua University cooperated with the 12th Institute of Electronic Industry to develop a complete $\mathrm{x}$-band standing wave accelerating tube because of funding of National Natural Science Foundation of China (No: 19075024), which promotes the development of Domestic xband small standing wave accelerator. Some other institutes were established by the colleges. For example, National Research and Engineering Center of Water-coal Slurry Gasification and Coal Chemical Industry established for supported by Yankuang Group Co., Ltd., East China University of Science and Technology, Tsinghua University and other colleges and universities.

Tsinghua University cooperated with 19 academic institutions to apply for 37 patents from 2000 to 2004. Institute of Telecommunication Transmission of Ministry of Information Industry account for the largest share and number of the cooperation patents are nine. Through information 
mining, we can find that the third generation mobile communication system of China was developed by Tsinghua University, Institute of Telecommunication Transmission of Ministry of Information Industry, and University of Science and Technology of China.

The cooperation patents have increased from 2005 to 2009. There are 37 academic institutions and 62 patents. The most of cooperation institute is Yangtze Delta Region Institute of Tsinghua University. It carries out the development model including the construction of government, industry, college, academic, financial, agency and user, which can improve the cooperation between local and colleges. This is the new cooperation model for regional government and university. Other institutions including Research Institute of Tsinghua University in Shenzhen, Information Technology Institute of Tsinghua University and China Hualu Group, China Research Center of Tsinghua University (Social Science) and Nomura Research Institute also follow this cooperation model. Otherwise, it is a common model that colleges and academic institutes assembled a research team to solve technical problems and realize scientific breakthrough. For instance, in order to research Quantum anomalous Hall Effect, researchers of Tsinghua University and Institute of Physics Chinese Academy of Sciences assembled joint research team.

There are 38 academic institutions cooperation with Tsinghua University and application 88 patents. Research Institute of Tsinghua University in Shenzhen accounts for the largest share. It shows that co-building research institutes is the effective way for Tsinghua University cooperation with local government.

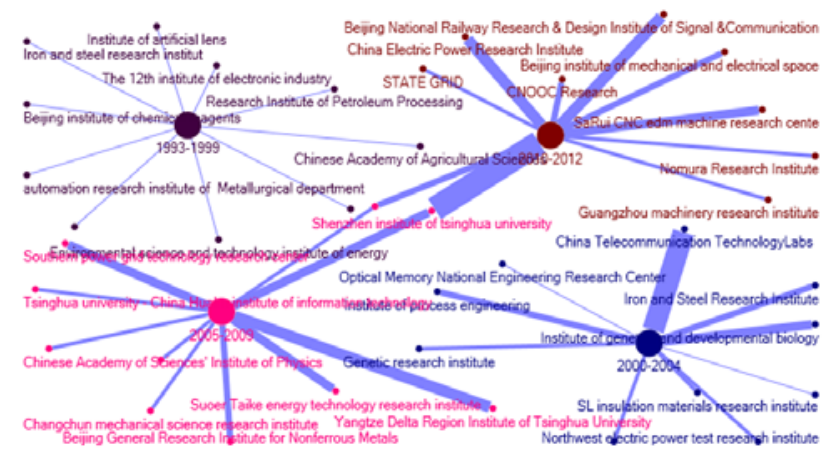

FIGURE II. Tsinghua University-Academic Institutions cooperation evolution path

\section{TSINGHUA UNI-ENTERPRISE COOPERATION EVOLUTION PATH}

Combination between colleges and enterprise has always been the important form of collaborative innovation, which is not only benefit technology innovation, but more beneficial to the products transformation of colleges innovation. Fig. 3 shows the patent cooperation situation between Tsinghua University and the top 10 enterprises in the fourth stage.

There are 21 academic institutions cooperation with Tsinghua University and 26 application patents from 1993 to 1999. Jingshang Qinghua Refrigeration Technology Co., Ltd, which is cooperative enterprise established by Japan Inoue corporation, Beijing Cheng-zhi Jin Technology Co., Ltd and
Tsinghua University, accounts for the largest share. The other two cooperation enterprises is Tsinghua Tongfang Co., Ltd and Beijing Tsinghua Yongchang Chemical Industry Co., Ltd which are new high-tech enterprise and directly controlled by Tsinghua University. In addition, cooperation way with Tsinghua University and other enterprises is joint development of technology. For instance, Haiyang Handicrafts Group Company cooperates with Tsinghua University to develop Fulda energy-saving heater.

There are 85 academic institutions cooperation with Tsinghua University to apply 289 patents from 2000 to 2004. The top three cooperation enterprises are Tsinghua Tongfang Co., Ltd, CapitalBio Cooperation and Hong Fujin Precision Industry Co., Ltd in Shenzhen. Tsinghua Tongfang Co., Ltd is controling by Tsinghua University and one of the most successful cases to promote synergy creation of production and transformation of scientific \& technological achievements. CapitalBio Cooperation is built to be jointly funded by Tsinghua University, Chinese Academy of Medical Sciences and other two institutes. It is different model from transplantation and risk investment of Tsinghua Tongfang Co., Ltd. The cooperation modern between Tsinghua University and CapitalBio Cooperation is Enterprise-Experiment mode that the enterprises are taken as the main part and the university is taken as technology support. Hong Fujin Precision Industry Co., Ltd in Shenzhen is a research and development company that Foxconn invested in the mainland. The cooperation is derived from the establishment of Tsinghua University - Foxconn Nanotechnology Research Center in 2003. The center improves good cooperation for a long time to carry out the foundation research and application research of Nanotechnology.

There are 126 academic institutions cooperation with Tsinghua University to apply 1009 patents from 2005 to 2009. The top three cooperation enterprises are Nuctech Co., Ltd, Beijing Visionox Technology Co., Ltd and Hong Fujin Precision Industry Co., Ltd in Shenzhen. Beijing Visionox Technology Co., Ltd is high-tech enterprises base on Organic light-emitting display technology of Tsinghua University, which set up in Beijing and Kunshan respectively. Beijing Ecore Technology Co., Ltd and Guodian Nanjing Automation Co., Ltd accounts for the larger share. Beijing Ecore Technology Co., Ltd is established by graduates of Tsinghua University .The most members of technology term are excellent Tsinghua University graduates. Moreover, in order to promote power system automation technology, China Guodian Corporation and Tsinghua University are joint to establish power system dispatching automation research institute of Tsinghua University and Guodian Nanjing Automation Co., Ltd.

There are 183 academic institutions cooperation with Tsinghua University to apply 946 patents from 2010 to 2012. Nuctech Co., Ltd, Beijing Visionox Technology Co., Ltd and CapitalBio Cooperation account for the larger share. Except for the enterprises controlling by Tsinghua University, other enterprises including Chengde Wanlitong Group Co., Ltd and Japan IHI Cooperation are also cooperate with Tsinghua University. Chengde Wanlitong Group Co., Ltd and Tsinghua University joint to set up the Flow Cell Engineering Research Center in 2009 because they together undertake the vanadium 
battery project of National 863 Program of China. Other enterprises are just transient cooperation with Tsinghua University to technology development. For instance, Energy Conservation Research Center of Tsinghua University and Beijing Huanneng Co., Ltd develop the absorption cycle technology which is the first urban central heating technology in world. Therefore, the cooperation between Tsinghua University and enterprises has entered the stable and long-term cooperative innovation stage. It is a major collaborative innovation model that colleges and enterprises collaborate on undertaking projects firstly and then establishing the Joint Research Centre.

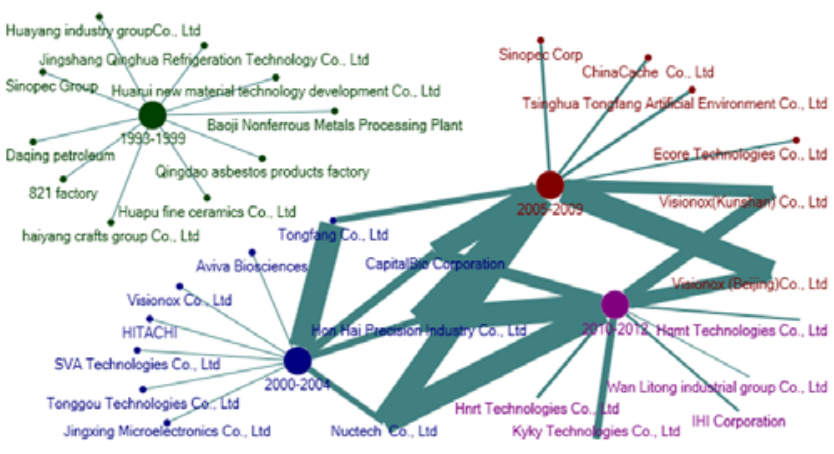

FIGURE III. Tsinghua Uni-enterprise cooperation evolution path

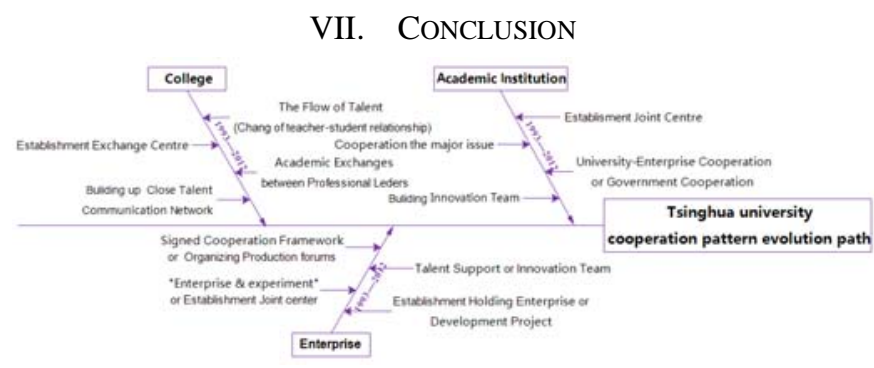

FIGURE IV

The evolution path of Tsinghua University cooperation model

Fig. 4 shows the evolution path of Tsinghua University cooperation model. According the results of data analysis and information mining, the process of cooperation model evolution is as follows.

(1)The cooperation between Tsinghua University and colleges: mainly rely on spontaneous collaboration between individual or small teams in recently 20 years. The cooperation model includes the change of relationship between teachers and students, joint training of talents, academic communication of professional leaders. Besides, Tsinghua University established communication center for the convenience of academic research and exploring new technology. Tsinghua University, which being a leading level of China in some technology fields, have established the stable and close communication network of technology, talents and academic exchange.

(2) The cooperation between Tsinghua University and academic institutes: The most of academic institutes rely on technology support of Tsinghua University. They are the product of strategic cooperation for talent cultivation, resource sharing and technical support between Tsinghua University and local government or enterprises. In addition, it is a common model that colleges and academic institutes assembled a research team to conquer technical problems or achieve scientific breakthrough.

(3) The cooperation between Tsinghua University and enterprises: Tsinghua University depends on the abundant talent resources and advanced technology to achieve a large number of scientific and technological innovation. And then Tsinghua University selected the industrialization of scientific and technological achievements as cooperation mode to build several high-tech enterprises which controlled by Tsinghua University. Since 2000, Tsinghua University and enterprises cooperate with Enterprise-Experiment mode that the enterprise is taken as the main part and the university is taken as technology support. Base on the cooperation, Tsinghua University and enterprises co-build research center, sign cooperation framework and hold academic forum.

\section{REFERENCES}

[1] Jianhua Hu. “Cooperation between Industry and Universities” Is the Key Approach to Collaborative Innovation of Universities: Taking Japan as an Example [J]. Journal of Nanjing Normal University (Social Science Edition),2012(5):30-37.

[2] Xiaoyan Liu,Pingnan Ruan,TongTong. Analysis of Influencing Factors of Knowledge Diffusion in Patent Cooperation Network-Integrated Circuits Industry as Example [J]. Forum on Science and Technology in China,2013(5):125-130,148.

[3] Ponomariov B. Government-sponsored university-industry collaboration and the production of nanotechnology patents in US universities[J]. Journal of Technology Transfer, 2013, 38(6): 749-767.

[4] James Simmie, Rolf Sternberg, Juliet Carpenter. New technological path creation: evidence from the British and German wind energy industries[J]. Journal of Evolutionary Economics, 2014, 24: 875-904.

[5] Feng-chao LIU, Rong-kang MA, Yu-tao SUN . Research on evolutionary path of nanotechnology based on technology co-occurrence network of patents $[\mathrm{J}]$. Studies in Science of Science, 2012,30(10):1500-1508.

[6] AIdong Peng, Huan Li,Yang Wang. Identifying of the technological evolution path based on patent citation network- laser display technology field as Example [J].Information Studies: Theory \& Application,2013,36(8):57-61.

[7] Janghyeok Yoon, Youngjin Park, Mujin Kim. Tracing evolving trends in printed electronics using patent information[J]. Journal of Nanoparticle Research, 2014, 16: 2471.

[8] Chan-Yuan Wong. On a path to creative destruction: science, technology and science-based technological trajectories of Japan and South Korea[J] Scientometrics,2013,96:323-336. 